SUBJECT AREAS:

MATERIALS SCIENCE

RENEWABLE ENERGY

GLASSES

NANOPARTICLES

Received

24 June 2014

Accepted

29 September 2014

Published

16 October 2014

Correspondence and requests for materials should be addressed to

R.A. (r.alghamedi@ ecu.edu.au)

\title{
Spectrally-selective all-inorganic
} scattering luminophores for solar energy-harvesting clear glass windows

\author{
Ramzy Alghamedi, Mikhail Vasiliev, Mohammad Nur-E-Alam \& Kamal Alameh
}

Electron Science Research Institute, Edith Cowan University, 270 Joondalup Drive, Joondalup, WA, 6027, Australia.

All-inorganic visibly-transparent energy-harvesting clear laminated glass windows are the most practical solution to boosting building-integrated photovoltaics (BIPV) energy outputs significantly while reducing cooling- and heating-related energy consumption in buildings. By incorporating luminophore materials into lamination interlayers and using spectrally-selective thin-film coatings in conjunction with $\mathrm{CuInSe}_{2}$ solar cells, most of the visible solar radiation can be transmitted through the glass window with minimum attenuation while ultraviolet (UV) radiation is down-converted and routed together with a significant part of infrared radiation to the edges for collection by solar cells. Experimental results demonstrate a $10 \mathrm{~cm} \times$ $10 \mathrm{~cm}$ vertically-placed energy-harvesting clear glass panel of transparency exceeding $60 \%$, invisible solar energy attenuation greater than $90 \%$ and electrical power output near $30 \mathrm{~W}_{\mathrm{p}} / \mathrm{m}^{2}$ mainly generated by infrared (IR) and UV radiations. These results open the way for the realization of large-area visibly-transparent energy-harvesting clear glass windows for BIPV systems.

T he role of renewable energy in addressing the challenges associated with implementing $\mathrm{CO}_{2}$ emissions reduction, addressing the climate change and energy supply concerns, has been recognised globally. Photovoltaics (PV), the conversion of sunlight to electricity has been reported to be the fastest-growing technology for electricity generation ${ }^{1}$. One-fifth of the world's total energy consumption is delivered for civil applications, residential and commercial ${ }^{2}$. Developing energy-efficient buildings is of prime importance, and future building industry regulations will have energy-efficiency requirements that meet the growing demand on energy resources. Ideally, new energy technologies must be integrated into the existing and future infrastructure elements (e.g. buildings), and at the same time provide savings by reducing the energy consumption. Designing "energy-saving and energy-producing solar windows" provides a way of boosting building-integrated PV (BIPV) energy outputs dramatically and beyond what is possible currently, simultaneously with reducing the coolingand heating-related energy consumption.

Various glazings and coated-type windows are in use worldwide as they offer thermal comfort improvements, energy savings, aesthetic value and ultraviolet (UV) protection. With the widespread use of glass panels covering vast areas of walls and roofs in modern buildings, significant economic benefits could be achieved if these glass surfaces had energy-generation capabilities. An economically-ideal energy-saving approach is to use window glazings that offer a combination of IR-range transparency blocking and energy-harvesting (due to the ability to efficiently convert the lighting-unrelated IR and UV solar energy into usable electric energy). It is likely that windows possessing these useful properties offer substantial economic and environmental benefits and would rapidly be recognized and marketed globally. It is well-known that industrial solar and wind farms require allocation of sizeable land areas in order to generate significant power. Conventionally, the power generation capability of green-energy PV installations is limited by the available roof area, especially for tall buildings. Wallmountable BIPV modules are starting to appear on the market, however, these mainly rely on thin-film photovoltaics of limited efficiency, and also cannot provide high optical transparency or energy savings related to solar-control properties.

Solar energy contains strong IR radiation flux coming from direct, diffused, reflected and re-emitted radiation from illuminated surfaces. Therefore, by using high-efficiency photovoltaic solar cells, energy-harvesting windows can generate measurable electric power even in non-ideal illumination conditions.

The development of principally new BIPV systems capable of high transparency and energy saving simultaneous with energy harvesting will bring the future goal of achieving net zero energy consumption in buildings closer to reality. The Luminescent Solar Concentrator (LSC) technology ${ }^{3-5}$ is currently being considered poten- 

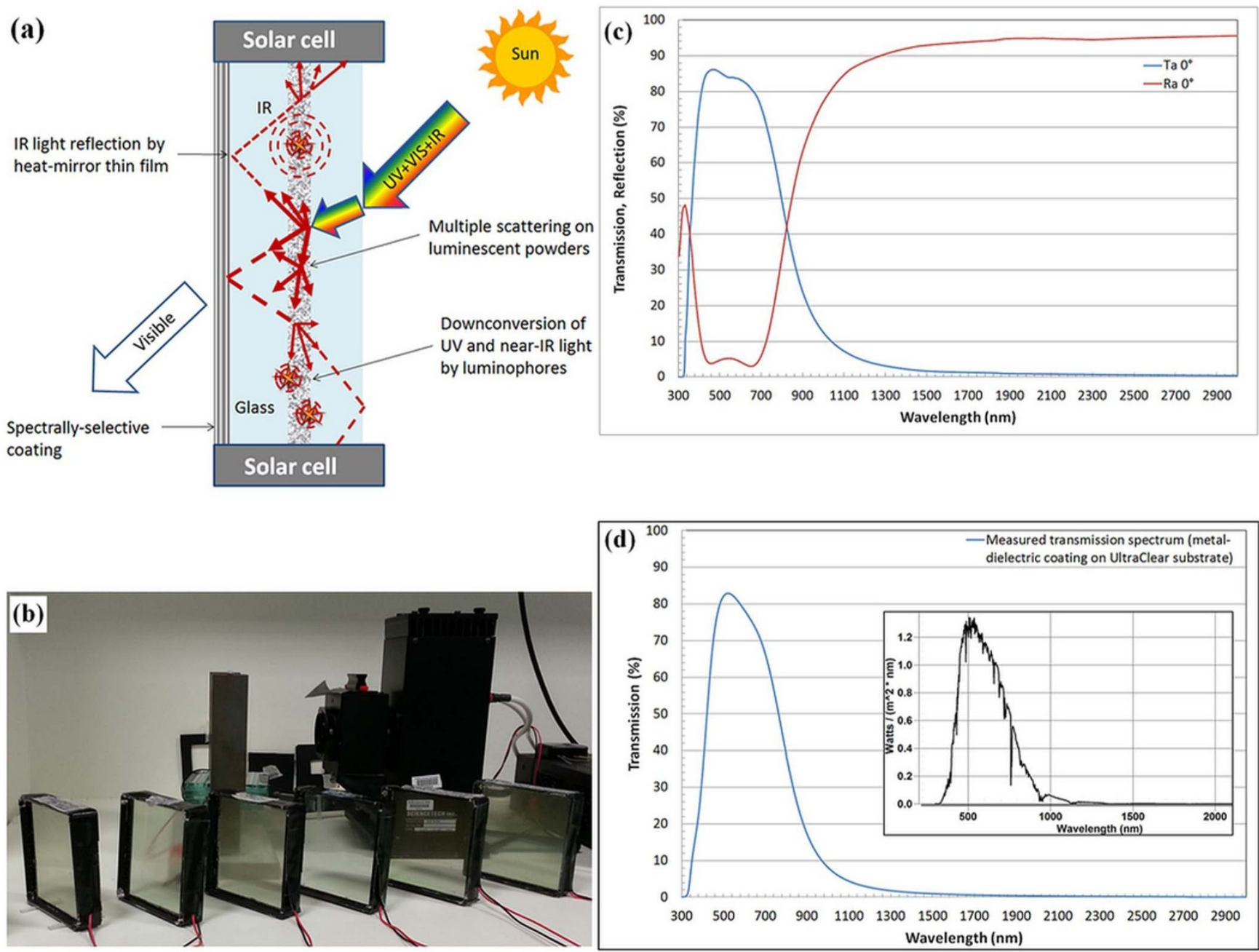

Figure 1 Concept and implementation of energy-saving and simultaneously energy-harvesting clear glass with its spectrally-selective solar-control coating. (a) Schematic of an energy-harvesting clear glass panel structure employing two low-iron (Starphire UltraClear) flat glass plates, a spectrallyselective multilayer coating deposited onto back surface of samples and luminescent interlayer containing inorganic luminophores incorporated into optical epoxy or resin-based lamination interlayers; (b) System implementation - several $100 \times 100$ mm energy-harvesting test samples with CuInSe ${ }_{2}$ (CIS) solar modules glued onto clear-cut panel edges. (c) Modeled transmittance and reflectance spectra of the optical interference coating, employing three different optical materials, designed to serve as a "heat mirror" and composed of two metal layers and five dielectric layers; (d) Measured transmission spectrum of the developed thin film (main graph), and the spectral power density distribution of the standard AM1.5G solar radiation transmitted through the coating-substrate system (inset).

tially suitable for engineering the PV windows of the future ${ }^{6}$, even though ensuring the high transparency in high-efficiency concentrators remains problematic for reasons of IR-luminophore limited availability. Organic dye-based LSCs have been employed to concentrate sunlight re-emitted at wavelength bands efficient for power generation by PV cells ${ }^{4,5,7,8}$. This type of concentrators commonly use dyes as luminescent organic species dispersed in optically clear polymer matrices ${ }^{7,8}$. However, it has been reported that when using organic dyes, the overlapping of absorption and emission bands reduces the concentration capabilities of LSC, which also contributes to their poor stability under long-term solar illumination exposure . $^{5}$ Moreover, most of LSC technologies and demonstrator samples featuring PV conversion of light concentrated by planar structures reported in the literature to date $\mathrm{e}^{9,10}$, share the following major limitations that still prohibit product-level development of energy-harvesting windows and at the same time emphasize the need for further research in this field:

- The concentrator-area scalability of all current LSC technologies is severely limited by the effects of re-absorption that reduce the propagation path-length of luminescence emissions within lightguiding structures;

- No LSC structures reported so far in the literature were designed to enhance the visible transparency simultaneously with spectrally-selective harvesting of non-visible solar radiation using inorganic-only luminophores. This is despite the fact that over $50 \%$ of solar energy reaching the ground level is distributed spectrally outside the visible range.

- The best power conversion efficiency of LSC devices reported to date remains within $2-4 \%$ if using $\mathrm{Si}$ cells. The sizes of most recently-reported high-performing concentrator panels never exceeded $100 \mathrm{~mm} \times 100 \mathrm{~mm}^{10,11}$.

To the best of our knowledge, no literature sources reported on achieving these $2-4 \%$ efficiency figures in samples of significant visible-range transparency, and most luminophores used have either been not disclosed or their compositions implied a limited environmental stability $^{9-11}$. Also, no spectrally-selective IR-specific photon-trapping structures suitable for use in window systems or glazing-type applications have ever been reported. 
Glass structure, technology and materials. A window design reflecting and redirecting the invisible solar radiation (Fig. 1(a)) exhibits LSC capability that relies on the efficient absorption of UV and IR light across as broad spectral range as is possible. This can be achieved using an active luminescent functionalized interlayer that provides photo-induced re-emission of the absorbed photons. The glass structure is integrated to act as spectrally-selective concentrator in such a way as to provide efficient transport of the re-emitted and otherwise internally deflected photons towards its edges.

Our solar concentration approach simultaneously enables electric power generation through PV cells attached to glass panel edges and maintains a maximized visible-range (400-700 nm) transparency. Concentrators of this type can be called "hybrid", since luminescence effects play only a partial role in energy harvesting functionality, together with multiple scattering and diffraction enabled by embedding quasi-random disordered arrays of luminophore particles inside polymer interlayers. Despite its contributions to optical losses within the concentrator structure ${ }^{12}$, multiple scattering effects can enhance light-trapping performance in planar-geometry structures $^{13-15}$. Our approach to the development of transparent hybridtype solar concentrators relies on the experimental optimization of electric energy output generated by the solar cells attached to the structure edges, by trialing a large number of luminescent material combinations. Some of these materials are presented in Table 1 and some others cannot be disclosed due to the confidentiality agreements. Generally, these materials are capable of converting the non-visible solar energy into near-IR emissions. Different ways of distributing the luminescent powders geometrically within interlayers have been trialed, including varying the particle sizes, epoxy loading concentrations, and layer thicknesses. Since a rather complex combination of several inter-related physical mechanisms and effects acting simultaneously was expected to lead (at least statistically) towards the increased IR illumination levels at sample edges, we chose to characterize the resulting structures' performance in terms of the actual energy harvesting output observed, in both the outdoor and solar-simulator experiments. Using PV cells attached to the sample edges was in this case the best practical way of detecting and measuring the total integrated contribution from all possible light redirection pathways present within the system structures, through measuring the electric output parameters such as open-circuit voltage $\left(\mathrm{V}_{\mathrm{oc}}\right)$ and short-circuit current $\left(\mathrm{I}_{\mathrm{sc}}\right)$. The relative contributions of different physical effects e.g. luminescence-assisted energy conversion versus multiple scattering or multiple diffraction events on powder particles is subject to our ongoing study.

A thin film interference-coating was especially designed to achieve minimized transmission of UV and IR energy as well as fulfil durability requirements related to environmental-stability. Fig. 1(c, d) shows the modelled and measured transmission and reflection spectra of a metal-dielectric thin film, which was developed using an ebeam/thermal evaporator. The heat-mirror-film design was adjusted in terms of materials selection and number of layers deposited to ensure wide-band reflection of IR radiation and the associated ther- mal insulation performance. Multiple coatings of this type were deposited onto glass substrates following the methods described in ${ }^{16}$.

Functionalized luminescent interlayers. To improve the light collection efficiency of light-trapping glass structures, we adopted an approach based on incorporating inorganic luminophore mixes into a transparent epoxy-based lamination layer, thus realizing a transparent, spectrally-selective, coating-assisted all-inorganic solar concentrator.

The selection of inorganic luminophore types for developing the interlayers as well as the optimization of powder particle sizes, concentrations, and interlayer thicknesses were subject to application constraints imposed by our industry partner (Tropiglas Technologies Ltd). The principal requirements were related to ensuring a substantial visible-range sample transparency and clear, non-colorbiased glass appearance. These requirements limited the selection of potential luminophores to inorganic substances possessing photoluminescence excitation ranges within either the UV or near-IR ranges, which could be ground into fine (of the order of $1 \mu \mathrm{m}$ ) particles, and then incorporated effectively by either dissolution or ultrasoundassisted disperse suspension, into the interlayer host material (epoxy). The required transparency and visual clarity of the functionalised interlayers were the variables that primarily controlled the upper limits of powder concentrations and interlayer thicknesses used. Prior to undertaking the research activities described in this paper, we had evaluated the relative energy-collection performance of a large number of $20 \times 20 \times 6 \mathrm{~mm}$ samples of similar structure, which employed different inorganic luminophore mixes of various concentrations and layer thicknesses as well as some luminescent organic dyes (Lumogen F Red and others). After extensive experimentation with multiple high-performance commercial photoluminophore substances from a range of suppliers, we identified four inorganic compositions from three different manufacturers which, when used individually or mixed together in small concentrations (sub-1 wt\% of each), led to substantial measured increases in the electric output from edge-mounted PV cells, when tested using a solar simulator beam directed at normal incidence (compared to a reference sample that did not contain luminophore materials within its epoxy-based interlayer). The beam of simulated sunlight was significantly smaller than the sample dimensions ( 2 "diam. vs $100 \mathrm{~mm}$ size), therefore some effective photon redirection mechanism within the glass structure (e.g. luminescence, Mie scattering promoting the wavevector deflection, diffraction events occurring on powder particles, or a combination of these) was crucial for increasing the photon flux reaching the side-mounted PV cells, as was evidenced by the significant dependency of electric output parameters on the interlayer type and composition. All samples constructed had interlayer thicknesses below $3 \mathrm{~mm}$, to ensure large visible transmission and good visual clarity, despite some noticeable scattering and diffused transmission effects. Here, we report on the empiric optimisation of the pre-selected functionalised interlayer compositions in $100 \times 100 \times 18 \mathrm{~mm}^{3}$ clear glass samples to maximise the electric power output in PV cells attached to structure edges

Table 1 | Luminescent material types used for interlayer functionalization

Phosphor ID Luminophore composition type

a $\quad \mathrm{Y}_{2} \mathrm{O}_{3}$ co-activated with several rare-earth ions and sensitized with $\mathrm{Yb}^{3+}$.

及 $\quad \mathrm{YPO}_{4}: \mathrm{Nd}$

$\gamma \quad(\mathrm{Zn}, \mathrm{Cd}) \mathrm{S}: \mathrm{Cu}$

$\delta$

$\mathrm{ZnS}:(\mathrm{Ag}, \mathrm{Tm})$
Excitation/Emission wavelength bands

Ex: mainly between $980 \pm 40 \mathrm{~nm}$
Em: $1000 \pm 50 \mathrm{~nm}$ and some visible upconversion emissions
excited by $980 \pm 40 \mathrm{~nm}$
Ex: $940-980 \mathrm{~nm}$
Em: near $1060 \mathrm{~nm}$
Ex: $300-550 \mathrm{~nm}$
Em: near $940 \mathrm{~nm}$
Ex: $300-430 \mathrm{~nm}$
Em: near $795 \mathrm{~nm}$




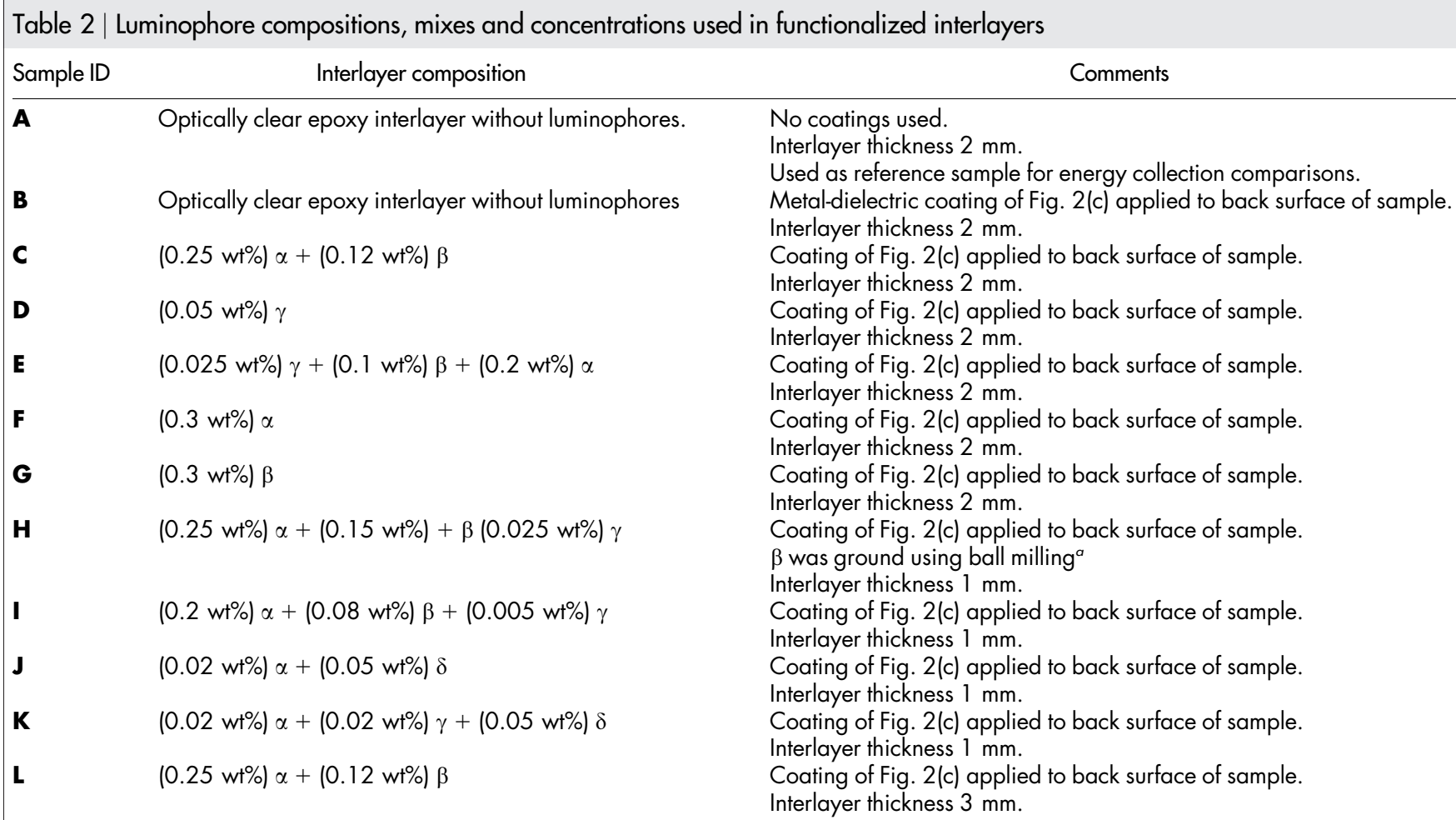

aPhosphor $\beta$ was normally dissolved into epoxy in unground state, except for sample H. Phosphor $\gamma$ was never ground since the particle dimensions in the powder available were between $0.5-3 \mu \mathrm{m}$. Phosphor $\alpha$ was always used after dry grinding for several hours using Fritsch Pulverisette Premium Line 7 ball mill at 700 rpm to reduce the mean particle size to the vicinity of $1-2 \mu \mathrm{m}$.

to detect and convert the optical power deflected towards the edges of glass structures. The effective path length of the luminescence excitation was difficult to evaluate, since the multi-pass propagation through the luminophore-loaded interlayer assisted by the multiple coating reflections and scattering events was engineered to occur within the structures.

Four different finely-ground luminophore powder types (described in Table 1) were selected (based on their performance characteristics such as excitation and emission spectral bands and quantum efficiency), and, in order to optimize the performance of the proposed energy-harvesting clear glass panel structure, various mixes were used to construct a number of concentrator prototypes of different interlayer chemistries.

The luminophore powders and their mixes were primarily selected to enable improved energy harvesting performance by providing both the UV, short-wave visible and also near-IR wavelength regions for photoluminescence excitation, and special care was also taken to place the emission wavelength regions inside the near-IR band, where the solar cells used had good responsivity, and also within the high-reflectance regions of the spectrally-selective coating. The function of $(\mathrm{Zn}, \mathrm{Cd}) \mathrm{S}$ : Cu luminophore $(\gamma)$ was to convert a fraction of light from (300-550) $\mathrm{nm}$ band into (840-1040) $\mathrm{nm}$. The main feature of this material and also of luminophore $\delta$, is related to the practical absence of overlapping between the excitation and emission wavelength bands, which effectively eliminates reabsorption-related energy losses. Emissions from all four phosphor types were within the high-responsivity band of the solar cell type used.

Partial concentrations of luminophores (in wt\%) were selected carefully in order to maintain the balance between optical clarity of the samples, reasonably high visible-range direct transmission, and enhancing the diffused transmission fraction together with material compatibility issues in terms of increasing scattering with increased number of powder types and concentrations used.

Several energy harvesting clear glass samples were assembled with different functionalized interlayer properties, such as luminophore compositions, concentrations and thicknesses as shown in Table 2. We carried out a large number of interlayer property adjustment experiments in order to keep the visible-range sample transparency above $40 \%$ while trialing the use of various simple, binary and ternary luminophore powder mixes and different loading concentrations to develop the most suitable interlayer material system for the routing of incident radiation towards solar cells.

Sample A of Table 2 was used as reference sample for benchmarking other samples' performance.

Sample testing methodology and energy harvesting performance characterization. We designed the concentrator and interlayer characterization experiments in order to identify the bestperforming samples (in terms of energy conversion and routing capability). The electric output parameters of samples were measured when illuminating the glass energy-collecting areas using a normally-incident, 2 " collimated (and also diffused) solar simulator beam spectrally equivalent to AM1.5G irradiation (Fig. 2(a)). A reference sample which intentionally did not incorporate any of the technology features designed to assist in energy routing towards glass edges, was selected for benchmarking the performance of all other samples. We define the "Area Collection Gain" parameter $(A C G)$ to characterize the relative flux-deflection capability in our concentrator samples as follows:

$$
A C G=\frac{\left(I_{s c, s a m p l e} * V_{o c, s a m p l e}-I_{s c, r e f} * V_{o c, r e f}\right)}{I_{s c, r e f} * V_{o c, r e f}} * 100 \%
$$

Parameter ACG (equation (1)) defines the way in which the reference sample is used and also describes the energy-routing performance differences between all samples measured through their electric output.

Figure 2(b) summarizes the results of performance testing experiments performed with all 12 concentrator samples each containing a different interlayer type. The $A C G$ measured in each sample varied depending on factors such as the luminophore chemistries used and 

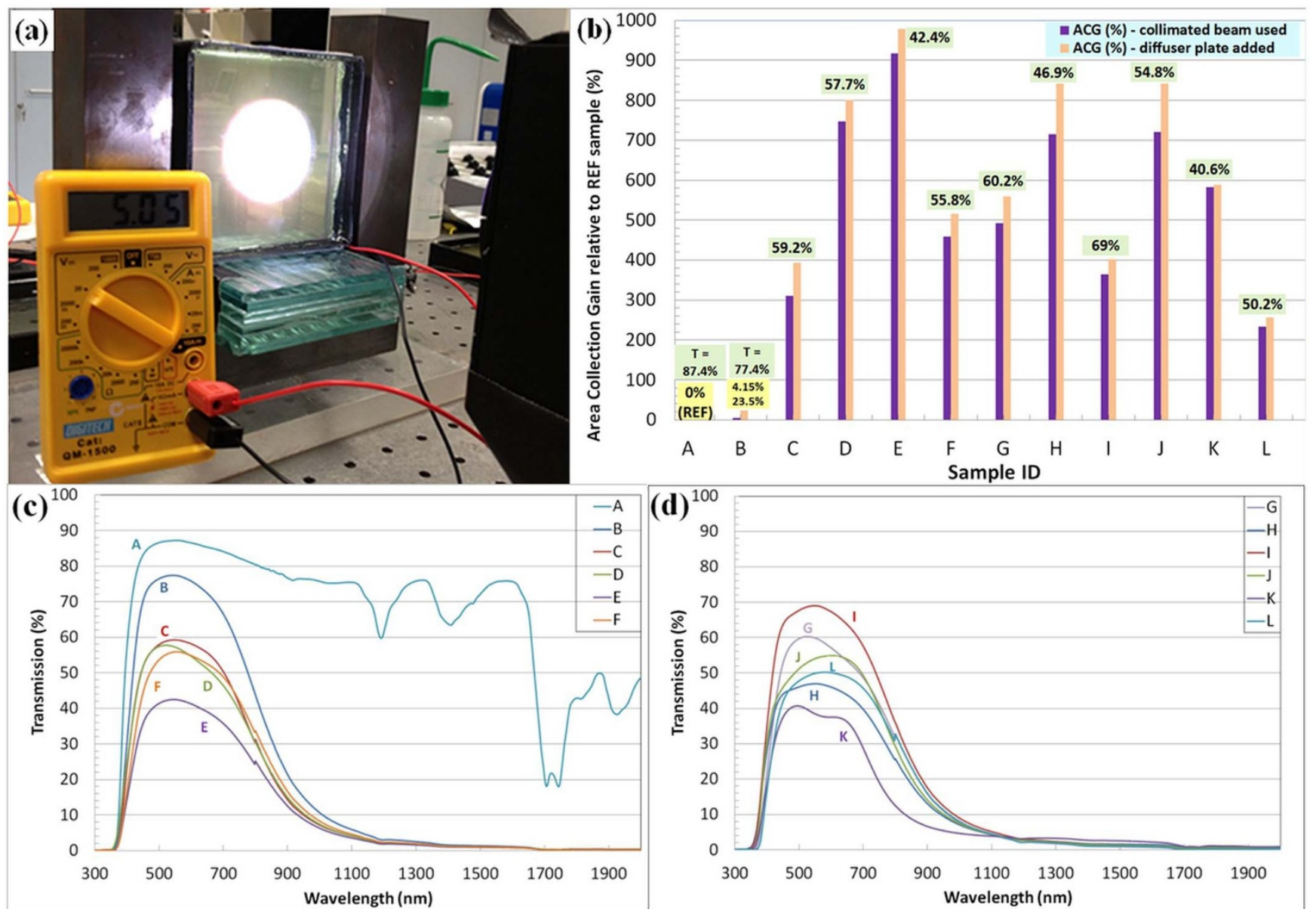

Figure $2 \mid$ Lab testing of the energy-harvesting capability of clear glass samples and performance characterisation results. (a) Sciencetech Inc. solar simulator used to direct a AM1.5G beam of 2" diameter towards the glass collector area of samples at normal incidence. The distance between sample glass surface and exit aperture of the solar simulator was kept constant for all samples; (b) ACG and peak visible transmission data measured in different samples; (c,d) measured transmission spectra of various energy-harvesting clear glass samples with different luminophore concentrations, material combinations and lamination layer thicknesses.

the concentrations of the powders, together with interlayer thickness. The goal was to search for an optimized interlayer type and properties, so as to deflect most of the non-transmitted incident optical power towards sample edges more effectively. For example, the results showed (as was somewhat expected) that the ability to deflect more radiation towards edges correlated inversely with visible transmission of samples. It is important to note that only the spectrum of direct transmission was measured, however diffuse transmission was also significant in our samples, as was evident from their visual appearance as well as from their light diffusion properties as illustrated in Fig. 3.

As an example, the functionalized interlayer of sample $C$ was made by mixing two luminophore powders $\alpha$ and $\beta$ the excitation bands of which overlapped significantly which, in conjunction with a relatively large scattering observed in all samples containing powder $\beta$, resulted in a relatively weak light collection performance. The optimization of light scattering intensity and the related light diffusion effects on the light collection efficiency and samples transmission was made only empirically in our experiments, since multiple factors affecting concentrator performance (from the varying solubilities of various powders in polymer materials to the final particle or agglomerate size ranges available from grinding and homogenization processes) could not be realistically predicted computationally. Sample $E$, on the other hand, contained three different powders which provided a combination of wide excitation bands placed in the $U V$, blue and also the near-IR wavelength regions, a rather large Stokes shift of luminophore $\gamma$, and the IR-range emission bands within the high responsivity wavelength range of CIS cells, showed considerably improved light concentration in comparison to most other samples. Luminophore $\gamma$ dissolved fairly well into our epoxies, had a rather
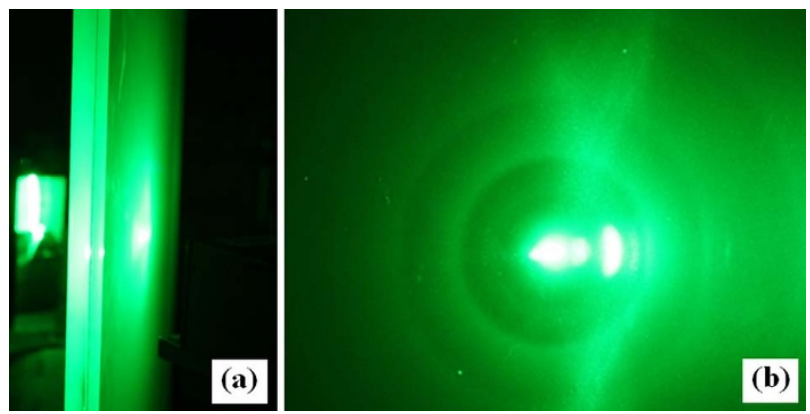

Figure 3 Light diffusion throughout the concentrator volume observed when illuminating a $200 \times 200 \mathrm{~mm}^{2}$ sample with a narrow (1-2 $\left.\mathrm{mm}\right)$ collimated beam from a $532 \mathrm{~nm}$ laser source at normal incidence.

(a) Diffused light rays reaching the sample's edge; (b) circular fringes of Young type observed within the diffraction halo formed across the glass panel surface originate from the interference of multiple scattered and diffracted rays propagating inside glass panel structure which contains a lamination interlayer loaded with a quasi-random distribution of luminophore particles. 

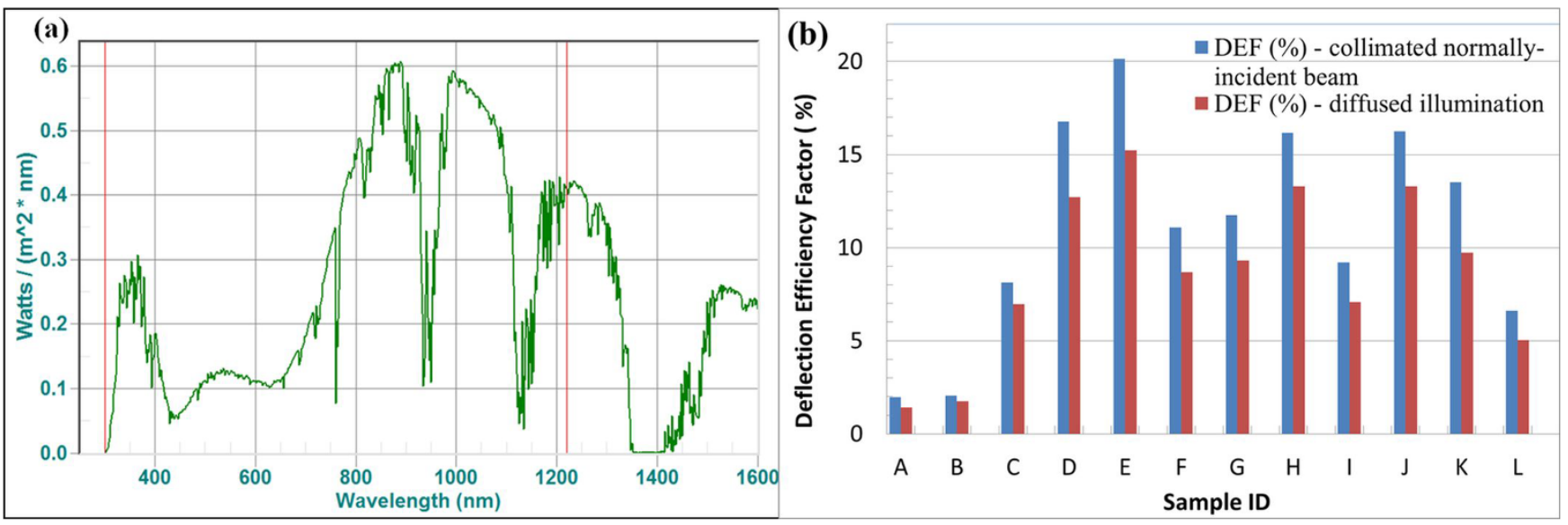

Figure $4 \mid$ Methodology for comparing the energy-harvesting performance of glass samples accounting for spectrally-selective separation of the harvestable part of the incoming solar radiation spectrum. (a) Spectral power density distribution of light reflected off spectrally-selective coating placed at the back surface of concentrator samples; (b) relative performance of different samples in terms of Deflection Efficiency Factor calculated using Eq.(3).

small mean particle size of about $1 \mu \mathrm{m}$, and did not cause significant scattering at concentrations not exceeding about $0.05 \mathrm{wt} \%$. The addition of other luminophores to the same sample increased scattering and decreased direct transmission in the visible range, while increasing the diffused transmission component. As shown in Fig. 2(b), the same ACG trends were seen across all samples in our batch, when using either the collimated-beam or diffused-beam illumination geometry.

The effects of multiple scattering on weakly-absorbing luminophore powder particles distributed in a quasi-random, quasi-3D fashion inside our interlayers, on the character of light propagation through concentrator samples are rather complex and lead to an additional scattering-related loss mechanism. However, the disorder-induced light trapping also occurs, and the possible light-trapping mechanisms involving multiple scattering effects in disordered photonic systems were reported recently in ${ }^{13-15}$. Additionally, the potential role of scattering effects in assisting the light trapping functionality in LSC systems requiring only modest flux gains has been recognized as early as $1981^{5}$. Fig. 3 shows that beam expansion and light diffusion effects are easily observable, as well as the appearance of fringe structure within a halo of diffused light formed due to multiple scattering on powder particles.

The disorder-induced beam expansion effects and their role in assisting the light trapping in planar hybrid-type optical concentrators is subject to our ongoing study.

The evaluation of the performance of LSC-type light concentration systems is commonly related to geometric flux gain measurements as well as to the measurements of the power conversion efficiency (overall system efficiency $\eta)^{4,5,10,11}$. For concentrator systems designed to provide significant spectral selectivity and thus spectrally separating the energy-harvesting wavelength bands from the enhanced-transmission bands, the definition of flux gain can be adjusted to account for the limited spectral bandwidth available for radiation routing and flux concentration. Parameters measurable directly in all PV systems include the short-circuit current $\left(I_{s c}\right)$ or its density $J_{s c}$, open-circuit voltage $\left(V_{o c}\right)$ and/or their product, or the output power $I_{s c} * V_{o c}{ }^{*} F F$. Within the framework of our experimental methodology, we define another figure of merit to characterize the energy routing (flux deflection) performance of concentrator samples assisted by spectrally-selective coatings, namely the Deflection Efficiency Factor $(D E F)$, in the following way:

First, we calculate the optical power (within the response bandwidth of PV cells used) of the normally-incident optical power, after the first coating-assisted reflection, that is

$$
P_{\text {refl,opt }}=A_{\text {beam }} * \int_{\lambda_{\min }}^{\lambda_{\max }} S_{A M 1.5 G}(\lambda) * R(\lambda) d \lambda
$$

Calculations according to equation (2) with the integration limits between $300 \mathrm{~nm}$ and $1220 \mathrm{~nm}$ show that the total optical power within the bandwidth of interest (for circular incident beam of 2 " diameter) after its first coating reflection $\left(P_{\text {reflopt }}\right)$ was only a small fraction $(26 \%)$ of the incident power.

Figure 4(a) shows the effect of our highly-transparent spectrallyselective solar-control coating on the spectral modification of the spectral power density distribution of incident light after the first reflection off the coating. We integrated the product of standard AM1.5G spectral distribution and the coating's reflectivity function numerically.

Second, we calculate the $D E F$ as follows:

$$
D E F=\frac{I_{s c}{ }^{*} V_{o c}{ }^{*} F F / \eta_{P V}}{A_{\text {beam }} * \int_{\lambda_{\min }}^{\lambda_{\max }} S_{A M 1.5 G}(\lambda)^{*} R(\lambda) d(\lambda)} * 100 \%
$$

This Deflection Efficiency Factor $(D E F)$ quantifies the optical power within the spectral responsivity band of CIS cells that has actually reached the cell surfaces, as a fraction of the optical power within the same spectral band, within the incident-beam area, available after the first reflection off the spectrally-selective coating placed at the back surface of the samples. This definition emphasizes the capability of the samples to deflect the energy available, accounting for the effects of transmission, scattering and absorption which limit the possible energy flux available for trapping or re-direction within the structure of the samples and interlayers. This way of evaluating the performance of the concentrator samples can be used for all types of PV cells, coatings and illumination conditions used, as long as the beam is centered within the glass collection area at near-normal incidence conditions. Figure 4(b) shows a summary of the relative performance of our energy harvesting clear glass samples in terms of $D E F$ as calculated using equation (3). Not surprisingly, significant dependency of $D E F$ on the interlayer type was observed.

\section{Principal Results and Discussion}

As was expected, the radiation deflection performance trends quantified using the DEF (Fig. 4(b)) followed closely the trends observed in the $A C G$ measurement results shown in Fig. 2(b). Sample $E$ 


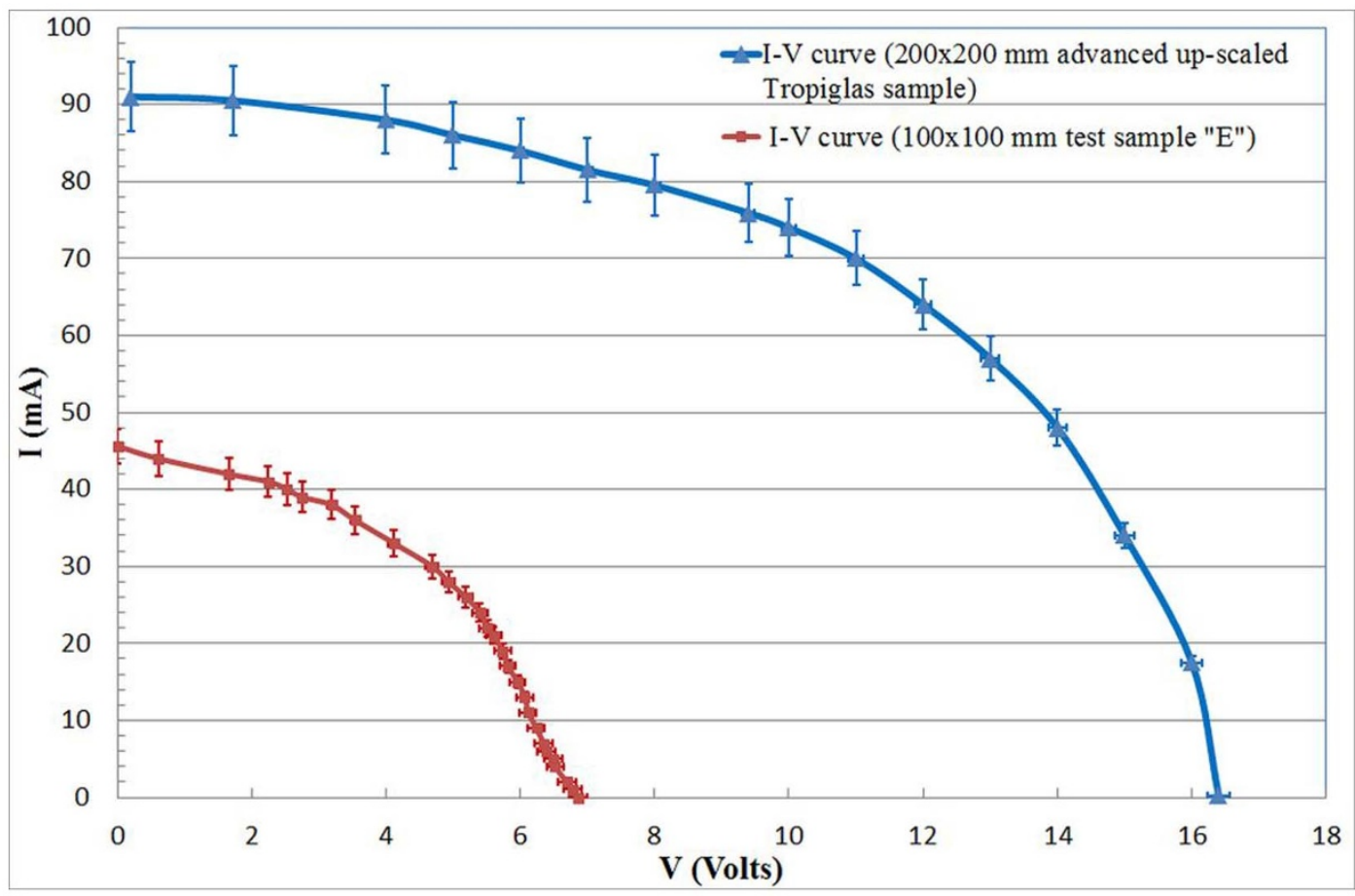

Figure $5 \mid$ Photovoltaic characterization results. I-V curves of one of the optimized up-scaled $200 \times 200 \mathrm{~mm}$ energy harvesting clear glass samples measured at peak outdoor illumination conditions, and of $100 \times 100 \mathrm{~mm}$ sample (E) measured outdoors during average insolation conditions. Both glazing system samples were oriented vertically during measurements.

showed a somewhat remarkable result of routing in excess of $20 \%$ of the total radiation energy reflected off its back coating towards the PV modules, considering that the input radiation was incident normally and multiple scattering and internal reflection events were required to propagate the photons incident onto the central region of glass collection area towards sample edges over a path length of several centimeters. In addition, the $V_{o c}$ generated by this sample $(5.12 \mathrm{~V})$ was approaching its saturation value (near $7.4 \mathrm{~V}$ for CIS modules of $100 \mathrm{~mm}$ length) despite not being directly illuminated by the light source.

Performance comparisons were also made in outdoor illumination conditions using vertically-oriented glass concentrator samples. The performance differences between the different sample types (within this batch of $100 \times 100 \mathrm{~mm}$ samples) were less pronounced compared to the results presented in Figs. 2 and 4, due to the increased direct (background) illumination reaching the CIS active areas, and the fact that the total area of CIS cells was as large as the glass collection area (which was in fact a design consideration for increasing the electric output of samples in lab conditions, where the background illumination of cells was quite easy to avoid), for reducing the $A C G$ and $D E F$ measurement errors.

Somewhat surprisingly, sample $J$ showed the best performance in peak outdoor illumination conditions $\left(I_{s c}=60.5 \mathrm{~mA} ; V_{o c}=7.4 \mathrm{~V}\right.$ and thus $\left.P_{\text {out }}=268.6 \mathrm{~mW}\right)$. When placed vertically, all samples intercepted a total flux of about $700 \mathrm{~W} / \mathrm{m}^{2}$ (7 W incident power), which brings the power conversion efficiency to $\eta=3.8 \%$. We believe that this result is outstanding for a glazing system-based concentrator of 55\% visible transparency employing inorganic-only luminophore combination. The cells were able to partially collect some background solar illumination in addition to collecting the concentrated light from inside the glass structure. Since approximately $50 \%$ of the air-exposed cell areas (at top and one side of sample, which accounted for $14 \%$ of the total CIS area) were essentially shadowed from direct sunlight, and the other exposed cell areas received only a fraction of incident flux due to geometry, most of the output was generated through concentration effects.
The results of interlayer optimization were used successfully to build up-scaled visibly-transparent glazing system concentrators of size $200 \times 200 \mathrm{~mm}$, however the detailed description of concentrator up-scaling results are beyond the scope of this article and will be reported separately. Fig. 5 shows typical I-V curves of our $100 \times$ $100 \mathrm{~mm}$ and advanced $200 \times 200 \mathrm{~mm}$ samples measured at "average" and peak outdoor illumination conditions, respectively.

The performance characteristics of our energy-harvesting glass samples quantified in terms of the parameters used commonly in the LSC field (such as power conversion efficiency $\boldsymbol{\eta}$, optical collection probability $P$ defined by $\boldsymbol{\eta} / \eta_{0}$ as a ratio of wall-plug device efficiency to bare-cell efficiency, and geometric gain $G$ [11]) are summarized in Table 3 . All of $100 \times 100 \mathrm{~mm}^{2}$ samples had a small geometric gain of near unity $(G=1.02)$, whereas the up-scaled $200 \times$ $200 \mathrm{~mm}^{2}$ sample had $G=1.87$.

The wall-plug (power conversion) efficiency and photon collection probability of sample $J$ both increased by almost $50 \%$ compared to sample $\boldsymbol{B}$ which relied on a blank (luminophore-free) epoxy interlayer and an identical structure and heat-mirror coating, due to adding an optimized concentration and mix of functional luminescent materials into its interlayer structure. Notable differences in the electric output stability versus the horizontal-angle orientation of these two samples were also seen, which were expected, because LSCtype collectors have an advantage of relative insensitivity to the incoming radiation flux direction. It is important to also note that, despite photoluminescence effects, other physical mechanisms including Mie scattering, multiple diffraction on a disordered array of powder particles and refractive-type coating-assisted geometric concentration, also contributed to energy collection at cells. The detailed analysis of relative contributions of all mechanisms leading to radiation flux re-direction and energy harvesting in visually-clear glass concentrators are subject to our ongoing studies.

\section{Conclusion}

We have proposed the use of all-inorganic spectrally-selective scattering luminophores in conjunction with spectrally-selective thin- 
Table 3 | Power conversion efficiency, optical collection probabilility, and optical concentration data obtained from several $100 \times$ $100 \mathrm{~mm}^{2}$ and one $200 \times 200 \mathrm{~mm}^{2}$ samples in peak outdoor illumination conditions

\begin{tabular}{|c|c|c|c|c|}
\hline Sample ID & $\eta$ & $P$ & $C$ & Comments \\
\hline$J$ & $3.8 \%$ & $41.3 \%$ & 0.41 & $\begin{array}{l}\text { Peak output current } I_{s c} \text { between } 55-60.5 \mathrm{~mA} \text { observed over a } \\
\text { very wide range of sample orientations (within } \pm 45-60^{\circ} \text { of } \\
\text { horizontal-plane angle changes). }\end{array}$ \\
\hline $\boldsymbol{E}$ & $3.55 \%$ & $38.63 \%$ & 0.386 & $\begin{array}{l}\text { Peak output current } I_{s c} \text { between } 50-56 \mathrm{~mA} \text { observed over a very } \\
\text { wide range of sample orientations (within } \pm 45-60^{\circ} \text { of } \\
\text { horizontal-plane angle changes). }\end{array}$ \\
\hline
\end{tabular}

film coatings and $\mathrm{CuInSe} e_{2}$ solar cells to realize visibly-transparent energy-harvesting clear laminated glass panels. Luminophore chemistries and concentrations have been optimized and incorporated into an optical epoxy lamination interlayer to maximize the power conversion efficiency of a $100 \mathrm{~mm} \times 100 \mathrm{~mm}$ vertically-placed energy-harvesting clear glass panel. Experimental results have demonstrated a transparency exceeding 55\%, invisible solar energy attenuation greater than $90 \%$, power conversion efficiency of $3.8 \%$, a solar heat gain coefficient (SHGC) of 0.41 , and a U-factor less than $1.8 \mathrm{~W} /\left(\mathrm{m}^{2} \mathrm{~K}\right)$.

\section{Methods}

Thin film coatings. Metal-dielectric coatings were manufactured by physical vapor deposition (PVD) techniques using sputtering targets and pellets of $99.99 \%$ pure materials purchased from AJA international, Inc. Chemically ultrasonically cleaned low iron borofloat glass cut-outs of size $100 \times 100 \times 6 \mathrm{~mm}$ were used as substrates. (KVE-ENT $200 \mathrm{hl}$ ) thermal/e-beam evaporator and (KVS-T4065) sputtering system, supplied by Korea Vacuum Tech, LTD, were used to deposit thin film layer sequences. The desired thin film characteristics were predicted using OptiLayer software package. The thin films transmission spectra were characterized using Agilent Cary 5000 UV-VIS-NIR and Beckman Coulter DU 640 B UV/Visible spectrophotometers.

Functionalized interlayer formation. The laminated glass functional interlayer composites were made with various concentrations and combinations of luminophore powders as shown in table 1 and table 2. NOA61 UV-curable epoxy (Norland, Inc.) and WTS-80203 (H.K. Wan Ta Shing, Industrial Ltd.) were used as polymer host matrices. No significant epoxy performance differences were noted when using these epoxies interchangeably.

Powders formed partially dissolved suspensions within the epoxy materials and were dispersed ultrasonically after mechanical stirring to de-agglomerate the suspended particles. Sonication of 100-150 ml epoxy volumes was performed for 5-10 minutes using Bandelin Sonopuls HD2200 fitted with $12 \mathrm{~mm}$ stainless steel horn, running at $10-20 \%$ of its transducer power. Interlayers were formed by pouring luminophore-loaded epoxy over glass sample surfaces manually. Layer thickness adapters were used at all sample corners during the liquid phase of the lamination process and then removed during layer solidification. Light curing system (ELC-410 Thorlabs, Inc.) was used as UV-blue light source for epoxy curing.

Solar cells integration and electrical circuits. $5 \mathrm{~W} \mathrm{CuInSe} e_{2}$ thin film solar cell modules of size $200 \times 270 \mathrm{~mm}$ on $2 \mathrm{~mm}$ - thick glass substrates (AD Solar, Inc.) were cut into strips of size $98 \times 25 \mathrm{~mm}$ using diamond-wheel glass cutter. The number of p-n junctions integrated along the cut-out lengths had to be made identical which was confirmed by making $I_{s c}$ and $V_{o c}$ measurements at full illumination to ensure uniform electric performance characteristics. Soldering connections to tabbing wires were made using ultrasonic soldering iron (PVLED Technology, Ltd.) and SB-220 (SBond, Inc.) active solder compound. Clear UV-curable epoxy identical to the interlayer host material was used as adhesive to attach the solar cell modules to each of the four laminated sample sides. All four cell modules were connected electrically in parallel using blocking diodes, since significantly non-uniform illumination conditions were expected to affect the system performance during outdoor operation of samples placed vertically.

Device characterization. Indoor tests were performed using a solar simulator system (ScienceTech, Inc.) as described in Principal Results and Discussion. Care was taken to ensure that all samples were tested at normal incidence conditions at a fixed $(100 \mathrm{~mm})$ distance from the source's output aperture, and that the AM 1.5G beam was centered onto the glass surface. Background illumination level was minimized by using curtains and removing room lighting.

Outdoor tests were performed in natural daylight conditions, and I-V characteristics were recorded manually (point by point) using 3700 Series Programmable DC
Electronic Load (Array Electronic Co., Ltd.) after orienting the vertically-placed samples in the horizontal plane to obtain peak output. The "peak" conditions were observed typically for horizontal-plane sample orientation angles near the $45^{\circ}$ flux incidence condition with respect to the Sun azimuth direction.

1. Green, M. A. Third generation photovoltaics: advanced solar energy conversion (Springer Series in Photonics, Vol. 12, Springer, Berlin, 2006).

2. U.S. Department of Energy, International Energy Outlook 2013. http://www.eia. gov/forecasts/ieo/pdf/0484(2013).pdf, (2013) Date of access:04/03/2014.

3. Baranov, V. K. Use of luminescent concentrators as spectral elements. Sov. J. Opt. Technol., 58, 472-474 (1991).

4. Batchelder, J., Zewail, A. \& Cole, T. Luminescent solar concentrators. 1: Theory of operation and techniques for performance evaluation. Appl. Opt. 18, 3090-3110 (1979).

5. Batchelder, J., Zewail, A. \& Cole, T. Luminescent solar concentrators. 2: Experimental and theoretical analysis of their possible efficiencies. Appl. Opt. 20, 3733-3754 (1981).

6. Meinardi, F., Colombo, A., Velizhanin, K. A., Simonutti, R., Lorenzon, M., Beverina, L., Viswanatha, R., Klimov, V. I. \& Brovelli, S. Large-area luminescent solar concentrators based on 'Stokes-shift-engineered' nanocrystals in a masspolymerized PMMA matrix. Nature Photon. 8, 392-399 (2014).

7. Evenson, S. A. \& Rawicz, A. H. Thin-film luminescent concentrators for integrated devices. Appl. Opt. 34(31), 7231-7238 (1995).

8. Evenson, S. A. \& Rawicz, A. H. Thin-film luminescent concentrators for integrated devices: a cookbook. Appl. Opt. 34(31), 7302-7306 (1995).

9. van Sark, W. G. J. H. M., Barnham, K. W. J., Slooff, L. H. et al. Luminescent Solar Concentrators - A review of recent results. Opt. Express 16(26), 21773-21792 (2008).

10. Debije, M. G. \& Verbunt, P. P. Thirty years of luminescent solar concentrator research: Solar energy for the built environment. Adv. Energy Mater. 2, 12-35 (2012).

11. Desmet, L., Ras, A. J. M., de Boer, D. K. G. \& Debije, M. G. Monocrystalline silicon photovoltaic luminescent solar concentrator with $4.2 \%$ power conversion efficiency. Opt. Lett. 37(15), 3087-3089 (2012).

12. de Boer, D. K. G., Broer, D. J., Debije, M. G., Keur, W., Meijerink, A., Ronda, C. R. \& Verbunt, P. P. C. Progress in phosphors and filters for luminescent solar concentrators. Opt. Express 20(S3), A395-A405 (2012).

13. Pratesi, F., Burresi, M., Riboli, F., Vynck, K. \& Wiersma, D. S. Disordered photonic structures for light harvesting in solar cells. Opt. Express 21(S3), A460-A468 (2013).

14. Sperling, T., Bührer, W., Aegerter, C. M. \& Maret, G. Direct determination of the transition to localization of light in three dimensions. Nature Photon. 7, 48-52 (2013).

15. Wiersma, D. S. Disordered photonics. Nature Photon. 7, 188-196 (2013).

16. El Mouedden, Y., Alghamedi, R., Nur-E-Alam, M., Vasiliev, M. \& Alameh, K. Thin film coatings for solar and thermal radiation control prepared by physical vapour deposition. in Proc. High Capacity Optical Networks and Enabling Technologies (HONET 2012, Istanbul, Turkey), pp. 083-086 (2012).

\section{Acknowledgments}

The authors would like to acknowledge the extensive support for this research provided by the Australian Research Council (ARC Linkage grant LP130100130), Tropiglas Technologies Ltd, Edith Cowan University, and the Saudi Arabian Cultural Mission in Australia and Taif University, Kingdom of Saudi Arabia.

\section{Author contributions}

The authors contributed equally to the review of research results and to the final presentation of this manuscript. The manufacture of solar energy harvesting glass samples included several preparation stages and component integration. The authors' contributions 
varied slightly during each of the stages. The design of thin film coating and running the deposition processes was mainly accomplished by R.A., M.V. and M.N. M.V. and M.N designed the electrical interconnections circuitry and performed the integration of solar modules. All four authors (R.A., M.V., K.A. and M.N.) worked on selecting the luminophore chemistries suitable for use in functionalised lamination interlayers and contributed to the fine-tuning of glass panels lamination processes. All authors participated in the design of both the indoor and outdoor solar energy harvesting performance characterisation experiments. The final data analysis was accomplished by R.A. and M.V. The figures and images included in the manuscript were generated throughout the research program, and all authors contributed actively to their contents. The research progress and the results achieved at each stage were supervised by K.A. from the intial stages; M.N. and K.A. reviewed the manuscipt after it had been written by R.A. and M.V., after which all authors again contrubuted to producing the final manuscript version.

\section{Additional information}

Supplementary information accompanies this paper at http://www.nature.com/ scientificreports

Competing financial interests: The authors declare no competing financial interests.

How to cite this article: Alghamedi, R., Vasiliev, M., Nur-E-Alam, M. \& Alameh, K. Spectrally-selective all-inorganic scattering luminophores for solar energy-harvesting clear glass windows. Sci. Rep. 4, 6632; DOI:10.1038/srep06632 (2014).

\section{()ㅇ $(\Theta \Theta$}

This work is licensed under a Creative Commons Attribution-NonCommercialNoDerivs 4.0 International License. The images or other third party material in this article are included in the article's Creative Commons license, unless indicated otherwise in the credit line; if the material is not included under the Creative Commons license, users will need to obtain permission from the license holder in order to reproduce the material. To view a copy of this license, visit http:// creativecommons.org/licenses/by-nc-nd/4.0/ 